\title{
ANÁLISE DOS ÓBITOS DE INFARTO AGUDO DO MIOCÁRDIO NO ESTADO DE SÃO PAULO
}

\section{ANALYSIS OF MYOCARDIAL ACUTE INFARCTION DEATHS IN THE STATE OF SÃO PAULO}

Emanoelle Aparecida Palangani ${ }^{1^{*}}$, Victor Barbosa Assis ${ }^{2}$, Yago Felyppe da Silveira Alvim Sant'ana ${ }^{3}$, Henry Ford Dal'col Frois ${ }^{4}$, Paulo César Silva Xavier ${ }^{5}$.

\author{
1 UNINGÁ - Centro Universitário Ingá, Maringá, PR, Brasil. \\ ${ }^{2}$ Universidade Federal da Paraíba, Cidade Universitária, PB, Brasil. \\ ${ }^{3}$ Universidade Federal de Juiz de Fora, Juiz de Fora, MG, Brasil. \\ ${ }^{4}$ Universidade Federal da Fronteira Sul, Laranjeiras do Sul, PR, Brasil. \\ ${ }^{5}$ Universidade de Santo Amaro, São Paulo, SP, Brasil. \\ *manupalangani@gmail.com
}

\section{RESUMO}

As doenças cardiovasculares, como a cardiopatia isquêmica, são um relevante problema de saúde pública no Brasil e no mundo, com altas taxas de incidência e mortalidade. Esse impacto pode ser explicado devido a modificação da estrutura etária populacional, e também a maior prevalência de exposição aos fatores de risco, tais como sedentarismo, aumento da prevalência da obesidade, tabagismo e aumento do consumo de uma alimentação não equilibrada. $O$ presente trabalho tem como objetivo, mostrar o estudo epidemiológico no estado de São Paulo relacionado às condições de vida que levam a internação por infarto agudo do miocárdio. Foi feito coleta e análise de dados do DATASUS, recorrendo ao intervalo entre 2010 a 2019. Como critérios de exclusão, foram retirados dados de pacientes com menos de 40 anos e de etnia indígena, pois faltavam informações ou apresentavam resultados irrelevantes para o estudo. Em consideração ao número de óbitos por infarto agudo do miocárdio no estado de São Paulo, que se totaliza em 23.998, foi possível destacar a predominância do sexo masculino $(56,09 \%)$, com 13.462 mortes, enquanto que o sexo feminino (43,90\%), apresentou 10.536. Com base na faixa etária, o intervalo de 40-49 anos (5,09\%), representou 1.223 óbitos; 50-59 (16,29\%), com 3.910 óbitos; 6069 (27,75\%), com 6.660 óbitos; $70-79$ (29,37\%), com 7.049 óbitos; e 80 anos e mais $(21,48 \%)$, apresentou 5.156 óbitos. Também foi analisado a influência da cor/etnia sobre essa condição, verificando que os pacientes brancos $(73,71 \%)$ foram mais acometidos por essa patologia, sendo 17.691 mortes; seguidos pelos pardos (21,73\%), com 5.217 mortes; e pacientes negros (4,54\%), com 1.090 mortes. Além disso, observou-se o aumento no número de óbitos por infarto agudo do miocárdio (37,54\%), onde no ano de 2010 ocorreram 2.083 mortes, enquanto que em 2019 aumentou para 2.865. Diante disso, é possível enfatizar o perfil epidemiológico das mortes associadas com essa patologia, sendo homens brancos, entre 70-79 anos, o grupo mais acometido. Assim como, o aumento da mortalidade, o que pode estar associado com as mudanças no estilo de vida da população e a carência da infraestrutura necessária para atender esses pacientes. Verifica-se a necessidade da criação de diretrizes clínicas adaptadas para os grupos de risco, com o intuito de realizar diagnósticos 
precoces e manejos mais efetivos, objetivando a redução da mortalidade no estado de São Paulo.

Palavras-chave: Cardiologia. Doenças cardiovasculares. Infarto do miocárdio. Isquemia Miocárdica. Pesquisas sobre Serviços de Saúde. 